\title{
Solution of the Hartree-Fock Equations for Atoms and Diatomic Molecules with the Finite Element Method
}

\author{
D. Heinemann and A. Rosén \\ Department of Physics, Chalmers University of Technology and University of Göteberg, S-41296 Göteborg, Sweden \\ and \\ B. Fricke
}

Department of Physics, University of Kassel, D-3500 Kassel, Federal Republic of Germany

Received February 27, 1990; and accepted in revised form June 5, 1990

\begin{abstract}
The finite element method (FEM) is now developed to solve two-dimensional Hartree-Fock (HF) equations for atoms and diatomic molecules. The method and its implementation is described and results are presented for the atoms $\mathrm{Be}, \mathrm{Ne}$ and $\mathrm{Ar}$ as well as the diatomic molecules $\mathrm{LiH}, \mathrm{BH}, \mathrm{N}_{2}$ and $\mathrm{CO}$ as examples. Total energies and eigenvalues calculated with the FEM on the HF-level are compared with results obtained with the numerical standard methods used for the solution of the one dimensional HF equations for atoms and for diatomic molecules with the traditional LCAO quantum chemical methods and the newly developed finite difference method on the HF-level. In general the accuracy increases from the LCAO - to the finite difference - to the finite element method.
\end{abstract}

\section{Introduction}

The one dimensional atomic problem on the HF-level has already been treated very early with great success in a purely numerical way [1]. With the access to powerful computers in the early sixties Herman and Skillman [2] published extensive calculations within the Hartree-Fock-Slater model for all elements in the periodic table. A good example of a relativistic atomic program using basis set expansion was set up by Kim [3]. The standard programs nowadays used to solve for atomic problems in Hartree-Fock (HF) or Multi-configuration Hartree-Fock (MCHF) [4] approximation or its relativistic counterparts (MCDF) [5, 6] all apply the one dimensional finite difference method. This holds also for the even more extended many Body Perturbation calculations (MBPT) [7, 8].

Until recently basis set expansion methods were the only ones available for two dimensional quantum chemical calculations which are based on the molecular orbital approach with a linear combination of atomic orbitals (MO-LCAO) [9]. Despite serious attempts the accuracy of such calculations on the HF level are rarely better than $10^{-5}$. The molecules which we are going to discuss here $\mathrm{LiH}$ and $\mathrm{BH}$ (Ref. [10]), $\mathrm{N}_{2}$ (Ref. [11]) as well as CO (Ref. [12]) as examples are all of this accuracy. Of course the absolute values of the total energies in the HF limit are by itself not so interesting but in order to get good values of the correlation contributions they are nevertheless of principal importance.

During the last years a number of computational schemes have been developed and tested for highly accurate numerical calculations for the solution of two dimensional problems in atomic and molecular physics. Such accurate methods are necessary to eliminate the basis set of truncation errors which are a serious bottleneck in quantum chemical calculations mentioned above. A review of the basis sets used in the quantum chemical calculations is given by Wilson [13]. The new developments are the two dimensional fully numerical finite difference method (FDM) [14] and the finite element method (FEM) [15]. It has been shown in both methods that the accuracy to calculate e.g., the total energy is many orders of magnitude better than in the old quantum mechanical method because both methods are basis set independent. With regard to the comparison of these two purely numerical methods the FEM seems to be even more stable and accurate than the FDM at least for the total energy. An overview of the finite difference method and a comparison with further numerical methods like the seminumerical partial wave method by McCullough [17] is given in Ref. [14]. Preliminary results for the solution of the $\mathrm{HF}$ equations with the FEM are given by Sundholm et al. in Ref. [15]. The papers published so far on the FEM from our group for the one- and twodimensional solution of the Hartree-Fock-Slater (HFS) and spin polarized HFS calculations are given in Ref. [16] and [18-21].

In Chapter 2 we are going to discuss in brief the HartreeFock problem for atoms and molecules as well as the FEM and computational approach. In Section 3 we discuss the results of the solution of the HF problem in a two dimensional way for the atoms $\mathrm{Be}, \mathrm{Ne}$ and $\mathrm{Ar}$ as well as $\mathrm{LiH}, \mathrm{BH}$, $\mathrm{N}_{2}$ and $\mathrm{CO}$ as examples for diatomic molecules.

\section{Theory}

\subsection{Hartree-Fock equations}

We repeat in short the basic HF equations [4] which we are going to solve in this paper with the FEM.

For a closed shell system the HF equations read

$$
\begin{aligned}
H_{\mathrm{HF}}(\boldsymbol{r}) \Phi_{i}(\boldsymbol{r}) & =\left(-1 / 2 \nabla^{2}+V^{\mathrm{nuc}}(\boldsymbol{r})+\sum_{j}\left(2 J_{j}-K_{j}\right)\right) \Phi_{i}(\boldsymbol{r}) \\
& =\varepsilon_{i} \Phi_{i}(\boldsymbol{r}) ; \quad i=1, \ldots, N
\end{aligned}
$$

Here $J_{j}$ and $K_{j}$ are the Coulomb and the exchange operators, respectively. $V^{\text {nuc }}(\boldsymbol{r})$ is the nuclear potential. The direct Coulomb operator $J_{j}$ can be written as

$$
\begin{aligned}
J_{j}(\boldsymbol{r}) \Phi_{i}(\boldsymbol{r}) & =\int \Phi_{j}^{*}\left(\boldsymbol{r}^{\prime}\right) \frac{1}{\left|\boldsymbol{r}-\boldsymbol{r}^{\prime}\right|} \Phi_{j}\left(\boldsymbol{r}^{\prime}\right) \mathrm{d} \boldsymbol{r}^{\prime} \Phi_{i}(\boldsymbol{r}) \\
& =V_{j}^{\mathfrak{c}}(\boldsymbol{r}) \Phi_{i}(\boldsymbol{r})
\end{aligned}
$$


whereas the exchange potential can be written

$$
\begin{aligned}
K_{j}(\boldsymbol{r}) \Phi_{i}(\boldsymbol{r}) & =\int \Phi_{j}^{*}\left(\boldsymbol{r}^{\prime}\right) \frac{1}{\left|\boldsymbol{r}-\boldsymbol{r}^{\prime}\right|} \Phi_{i}\left(\boldsymbol{r}^{\prime}\right) \mathrm{d} \boldsymbol{r}^{\prime} \Phi_{j}(\boldsymbol{r}) \\
& =V_{j i}^{\mathrm{x}}(\boldsymbol{r}) \Phi_{j}(\boldsymbol{r})
\end{aligned}
$$

The direct Coulomb potential $V_{j}^{\mathrm{c}}(\boldsymbol{r})$ satisfies the Poisson equation

$$
\nabla^{2} V_{j}^{\mathrm{c}}(\boldsymbol{r})=-4 \pi \rho_{j}(\boldsymbol{r})
$$

with

$$
\rho_{j}(\boldsymbol{r})=\left|\Phi_{j}(\boldsymbol{r})\right|^{2}
$$

and the exchange potential $V_{j i}^{\mathrm{x}}(\boldsymbol{r})$ the Poisson like equation $[22,23]$

$$
\nabla^{2} V_{j i}^{\mathrm{x}}(\boldsymbol{r})=-4 \pi \rho_{j i}(\boldsymbol{r})
$$

with

$\rho_{j i}(\boldsymbol{r})=\Phi_{i}^{*}(\boldsymbol{r}) \Phi_{j}(\boldsymbol{r})$

The actual solution of the HF equations is done in an iterative procedure. If we start with a set of approximations $\Phi_{j}^{\prime}(\boldsymbol{r})$ of the unknown wavefunctions $\Phi_{i}(\boldsymbol{r})$ we can calculate the direct Coulomb potential $V_{j}^{\mathrm{c}}(\boldsymbol{r})$ and the exchange potential $V_{j i}^{\mathrm{x}}(\boldsymbol{r})$. The expectation value of the one particle energy can be evaluated as

$\varepsilon_{j}=\left\langle\Phi_{j}^{\prime}\left|H_{\mathrm{HF}}\right| \Phi_{j}^{\prime}\right\rangle$

In order to determine the new approximation of the wavefunctions eq. (1) is rewritten to

$$
\begin{gathered}
\left(-1 / 2 \nabla^{2}+V^{\text {nuc }}(\boldsymbol{r})+2 \sum_{j} V_{i}^{\mathrm{c}}-\varepsilon_{i}\right) \Phi_{i}(\boldsymbol{r})=\sum_{j} V_{j i}^{\mathrm{x}} \Phi_{j}(\boldsymbol{r}) \\
i=1, \ldots, N
\end{gathered}
$$

using the definitions of eqs. (2) and (3). For the right hand side of eq. (7) the approximations $\Phi_{j}^{\prime}(\boldsymbol{r})$ of the wavefunctions $\Phi_{j}(\boldsymbol{r})$ are used. Thus the right hand side of eq. (7) is a known function and the equation can be solved for a new approximation of the wavefunction $\Phi_{i}(\boldsymbol{r})$. This can formally be written as

$$
\tilde{L}_{i} \Phi_{i}(\boldsymbol{r})=X_{i}(\boldsymbol{r})
$$

with

$\widetilde{L}_{i}=-1 / 2 \nabla^{2}+V^{\mathrm{n} w \mathrm{c}}(\boldsymbol{r})+V^{\mathrm{c}}(\boldsymbol{r})-\varepsilon_{i}$

and

$$
\begin{aligned}
V^{\mathrm{e}} & =2 \sum_{j} V_{j}^{\mathrm{c}}(\boldsymbol{r}), \\
X_{i} & =\sum_{j} V_{j i}^{\mathrm{x}}(\boldsymbol{r}) \Phi_{j}(\boldsymbol{r}) .
\end{aligned}
$$

Formally eq. (8) takes the same form as eqs. (4) and (5) and is solved by the FEM in a similar way. The Hartree-Fock iteration is started with wavefunctions calculated with the Thomas-Fermi potential of the system.

\subsection{Finite element method and computational approach}

The FEM method was originally developed in engineering science in order to calculate static and dynamic stresses of complicated constructions. Recently, it has been demonstrated [24-31, 35] that this method can also be used with great success to solve quantum mechanical problems.

In order to apply the FEM to the solution of the HF- equations one starts from the variational equivalent of the second order partial differential equations. Details of this formulation and the details of the computational method can be found in Ref. [14].

Using axial symmetric coordinate systems as defined in Ref. [14] the angular coordinate can be treated analytically as we write,

$$
\begin{aligned}
\Phi_{i}(s, t, \phi) & =\Phi_{i}(s, t) \exp \left(\mathrm{im}_{i} \phi\right) \\
V_{i}^{\mathrm{c}}(s, t, \phi) & =V_{i}^{\mathrm{c}}(s, t) \\
V_{j i}^{\mathrm{x}}(s, t, \phi) & =V_{j i}^{\mathrm{x}}(s, t) \exp \left(\mathrm{in}_{j i} \phi\right)
\end{aligned}
$$

$m_{i}$ denotes the projection of the angular momentum onto the internuclear axis of orbital $\Phi_{i}$ and $n_{j i}$ is the difference $n_{i i}=$ $\left(m_{i}-m_{j}\right)$ of the $m$-quantum numbers of the orbitals $\Phi_{i}$ and $\Phi_{j}$.

With these representations of the wavefunctions and potentials the variational integral of eq. (4) takes the form

$$
\begin{aligned}
I_{1}= & \int-\frac{1}{2} K_{1}\left(\frac{\partial V_{i}^{\mathrm{c}}}{\partial s}\right)^{2}-\frac{1}{2} K_{2}\left(\frac{\partial V_{i}^{\mathrm{c}}}{\partial t}\right)^{2} \\
& +4 \pi K_{4}(s, t) \rho_{i}(s, t) V_{i}^{\mathrm{c}} \mathrm{d} s \mathrm{~d} t
\end{aligned}
$$

The variational integral of eq. (5) can be written

$$
\begin{aligned}
I_{2}= & \int-\frac{1}{2} K_{1}\left(\frac{\partial V_{j i}^{\mathrm{x}}}{\partial s}\right)^{2}-\frac{1}{2} K_{2}\left(\frac{\partial V_{j i}^{\mathrm{x}}}{\partial t}\right)^{2}-\frac{1}{2} n_{j i}^{2} K_{3}\left(V_{j i}^{\mathrm{x}}\right)^{2} \\
& +4 \pi K_{4}(s, t) \rho_{j i}(s, t) V_{j i}^{\mathrm{x}} \mathrm{d} s \mathrm{~d} t
\end{aligned}
$$

and the integral of eq. (7) is

$$
\begin{aligned}
I_{3}= & \int-\frac{1}{4} K_{l}\left(\frac{\partial \Phi_{i}}{\partial s}\right)^{2}-\frac{1}{4} \mathbf{K}_{2}\left(\frac{\partial \Phi_{i}}{\partial t}\right)^{2}-\frac{1}{4} m_{i}^{2} K_{3}\left(\Phi_{i}\right)^{2} \\
& -\frac{1}{2}\left(V-\varepsilon_{i}\right) K_{4}\left(\Phi_{i}\right)^{2}-\frac{1}{2} X_{i} K_{4} \Phi_{i}(s, t) \mathrm{d} s \mathrm{~d} t
\end{aligned}
$$

here $V$ denotes the sum of nuclear and direct Coulomb potential. The coefficient functions $K_{1}, \ldots, K_{4}$ are calculated from the coordinate transformation functions of the coordinate system in use [16]

The next step in applying the FEM is to subdivide the two-dimensional space of our problem into $M$ small domains called elements. The subdivision of the space into elements can be adapted to the physical problem by combining triangles of different sizes and shapes. On each of the elements the unknown function $f(s, t)$ is approximated by a trial function $u(s, t)$ which is written as

$u(s, t)=\sum_{k=1}^{p} u_{k} N_{k}(s, t)$

a linear combination of the two-dimensional polynomials $N_{k}(s, t)$. These polynomials are defined such that the coefficients $u_{k}$ can be interpreted as the values of the unknown function $f(s, t)$ at certain points. In our case we choose two-dimensional Lagrange polynomials up to order 6 as trial functions. Because of the subdivision of the region into $M$ elements the functionals $I_{1}$ (eq. (15)) $I_{2}$ (eq. (16)) and $I_{3}$ (eq. (17)) can be written as a sum of element integrals

$$
I_{1}=\sum_{i=1}^{M} I_{1}^{e}, \quad I_{2}=\sum_{i=1}^{M} I_{2}^{e} \text { and } I_{3}=\sum_{i=1}^{M} I_{3}^{i},
$$

with integrals running over the areas of the $M$ elements. The substitution of the trial function $u(s, t)$ into the element 
integrals $I_{1}^{e}, I_{2}^{e}$ and $I_{3}^{e}$ and summation according to eq. (19) leads to matrix expression for the variational integrals.

$I_{1}=\boldsymbol{u}^{\mathrm{t}} \cdot \mathbf{D}_{i}^{\mathrm{c}} \cdot \boldsymbol{u}-\boldsymbol{u}^{\mathrm{t}} \cdot \boldsymbol{d}_{i}$

$I_{2}=\boldsymbol{u}^{\mathrm{t}} \cdot \mathbf{D}_{i j}^{\mathrm{c}} \cdot \boldsymbol{u}-\boldsymbol{u}^{\mathrm{t}} \cdot \boldsymbol{d}_{i j}$

$I_{3}=\boldsymbol{u}^{\mathrm{t}} \cdot \mathbf{L}_{i} \cdot \boldsymbol{u}-\boldsymbol{u}^{\mathrm{t}} \cdot \boldsymbol{X}_{i}$

As the global matrices $\mathbf{D}_{i}^{\mathbf{c}}, \mathbf{D}_{i j}^{\mathrm{c}}$ and $\mathbf{L}_{i}$ are built up by contributions of elements which are distinct in space they are band structured matrices. The vectors $\boldsymbol{u}$ contain the coefficients $u_{k}$ of eq. (18) from all elements. The functionals $I_{1}, I_{2}$, and $I_{3}$ can now be seen as functions of these coefficients. The conditions for $I_{1}, I_{2}$, and $I_{3}$ to be extreme therefore are

$\left(\frac{\partial I_{1}}{\partial u_{k}}\right)=0, \quad\left(\frac{\partial I_{2}}{\partial u_{k}}\right)=0, \quad\left(\frac{\partial I_{3}}{\partial u_{k}}\right)=0, k=1, \ldots, N_{\mathrm{p}}$.

These conditions lead to the matrix equations

$$
\begin{aligned}
\mathbf{D}_{i}^{\mathrm{c}} \cdot \boldsymbol{u} & =\boldsymbol{d}_{i} \\
\mathbf{D}_{i j}^{\mathrm{c}} \cdot \boldsymbol{u} & =\boldsymbol{d}_{i j} \\
\mathbf{L}_{i} \cdot \boldsymbol{u} & =\boldsymbol{X}_{i}
\end{aligned}
$$

The resulting matrix eqs. (24), (25) and (26) are solved by a Cholesky type decomposition of the matrices. After the solution of eq. (8) for the different wavefunctions of same symmetry due to the solution of the matrix eq. (26), the different vectors $\boldsymbol{u}$ are orthonormalized using Schmidt's orthonormalization method.

The boundary conditions for the Poisson type eqs. (4) and (5) are calculated by means of a multipole expansion of the densities $\rho_{i}(\boldsymbol{r})$ or the exchange densities $\rho_{i j}(\boldsymbol{r})$, respectively. As boundary values for the wavefunctions the value $\Phi_{i}(\boldsymbol{r})=0$ is used for practical infinity at distances of $25 \mathrm{a} . \mathrm{u}$.

\section{Results and discussion}

\subsection{Atoms}

The two-dimensional approach described above was tested by solving for the atoms $\mathrm{Be}, \mathrm{Ne}$ and $\mathrm{Ar}$ as examples. The calculations were performed with up to 385 points for $\mathrm{Be}, 931$ points for $\mathrm{Ne}$ and 1729 points for Ar. The results given in Table I are compared with the results of one-dimensional calculations $[4,32,33]$ in the HF limit where the last digit given is uncertain. The agreement between both methods is perfect.

Because the FEM calculation explicitly allows a two dimensional freedom for the wavefunctions they are thus two dimensional unrestricted HF calculations, which in principle allow for deformation in the second dimension. We started the FEM calculations with a dipole and quadrupole deformed initial potential, but during the convergence all three atoms calculated so far definitely converged towards the spherical symmetric restricted HF limit. This behaviour seems to be obvious because all these atoms are closed shell systems, but it is important to see that they really behave like this.

\subsection{Diatomic molecules}

The first results for diatomic molecules in the HF limit are given in Table II for the two hydrids $\mathrm{LiH}$ and $\mathrm{BH}$ and in Table III for the systems $\mathrm{N}_{2}$ and $\mathrm{CO}$ as typical examples for first row molecules. All these results are compared for the total energies and the energy eigenvalues of the occupied levels first with the best quantum chemical MO-LCAO calculations on the HF level and second with the finite difference calculations of Laaksonen et al. [14]. For all these systems we used 2401 points. In order to improve the accuracy the sizes of the elements were adjusted by a logarithmic scaling [21]. The last digit in the values given is uncertain.

For the system $\mathrm{LiH}$ the agreement with the value of Laaksonen et al. [14] is in the order of $10^{-7}$ a.u. for the

\begin{tabular}{|c|c|c|c|c|}
\hline & \multicolumn{4}{|c|}{$B e$} \\
\hline & This work & This work & This work & Ref. [4] \\
\hline Points & 175 & 301 & 385 & \\
\hline$E_{\mathrm{tot}}$ & -14.573019 & -14.57302316 & -14.57302317 & -14.573023 \\
\hline$\varepsilon(1 s)$ & -4.732674 & -4.732669898 & -4.732669897 & -4.732669897 \\
\hline$\varepsilon(2 s)$ & -0.3092695 & -0.309269551 & -0.309269552 & -0.30926955 \\
\hline \multicolumn{5}{|l|}{$\mathrm{Ne}$} \\
\hline & This work & This work & This work & Ref. [31] \\
\hline Points & 481 & 637 & 931 & \\
\hline$E_{\mathrm{tot}}$ & -128.547093 & -128.547097 & -128.547098 & -128.547098 \\
\hline$\varepsilon(1 s)$ & -32.772442 & -32.772443 & -32.772443 & -32.772443 \\
\hline$\varepsilon(2 s)$ & -1.930391 & -1.930391 & -1.9303909 & -1.9303909 \\
\hline$\varepsilon(2 p \pi)$ & -0.850410 & -0.8504097 & -0.85040964 & -0.8504096 \\
\hline$\varepsilon(2 p \sigma)$ & -0.850409 & -0.8504095 & -0.85040963 & -0.8504096 \\
\hline \multicolumn{5}{|l|}{$A r$} \\
\hline & This work & This work & This work & Ref. [33] \\
\hline Points & 403 & 949 & 1729 & \\
\hline$E_{\mathrm{tol}}$ & -526.8171 & -526.81751 & -526.8175127 & -526.8175130 \\
\hline$\varepsilon(1 s)$ & -118.61041 & -118.61036 & -118.6103506 & -118.6103506 \\
\hline$\varepsilon(2 s)$ & -12.32217 & -12.32216 & -12.3221533 & -12.3221533 \\
\hline$\varepsilon(2 p \pi)$ & -9.57153 & -9.57148 & -9.5714656 & -9.5714656 \\
\hline$\varepsilon(2 p \sigma)$ & -9.57149 & -9.57147 & -9.5714656 & -9.5714656 \\
\hline$\varepsilon(3 s)$ & -1.27736 & -1.277358 & -1.2773530 & -1.2773530 \\
\hline$\varepsilon(3 p \pi)$ & -0.59104 & -0.591023 & -0.5910174 & -0.5910174 \\
\hline$\varepsilon(3 p \sigma)$ & -0.59102 & -0.591021 & -0.5910174 & -0.5910174 \\
\hline
\end{tabular}

Table I. A comparison of total energy and eigenvalues for the atoms Be, Ne and Ar calculated with the two dimensional FEM using different grid sizes and one-dimensional $H F$ 
Table II. Total energy and molecular eigenvalues for the diatomic molecules $\mathrm{LiH}$ and BH calculated with the FEM using different grid sizes. Comparison is done with results of FDM calculations and LCAO basis set expansion methods

\begin{tabular}{|c|c|c|c|c|}
\hline \multicolumn{5}{|l|}{$\mathrm{LiH}$} \\
\hline Points & $\begin{array}{c}\text { This work } \\
625\end{array}$ & $\begin{array}{c}\text { This work } \\
1369\end{array}$ & Ref. [14] & Ref. [10] \\
\hline$R$ & 3.015 & 3.025 & 3.015 & 3.015 \\
\hline$E_{\mathrm{tot}}$ & -7.9873517 & -7.8973522 & -7.9873524 & -7.987313 \\
\hline$\varepsilon(1 \sigma)$ & -2.4452370 & -2.4452338 & -2.4452338 & -2.44525 \\
\hline$\varepsilon(2 s)$ & -0.3017360 & -0.3017382 & -0.3017383 & -0.30172 \\
\hline \multicolumn{5}{|l|}{$B H$} \\
\hline & This work & This work & Ref. [14] & Ref. $[10]$ \\
\hline Points & 1369 & 2401 & & \\
\hline$R$ & 2.336 & 2.336 & & \\
\hline$E_{\mathrm{zot}}$ & -25.1315984 & -25.1315987 & -25.131609 & -25.13137 \\
\hline$E(1 \sigma)$ & -7.6861673 & -7.6862674 & -7.686271 & -7.68633 \\
\hline$\varepsilon(2 \sigma)$ & -0.6481873 & -0.6481873 & -0.648188 & -0.64816 \\
\hline$\varepsilon(3 \sigma)$ & -0.3484238 & -0.3484238 & -0.348424 & -0.34837 \\
\hline
\end{tabular}

total energy whereas the traditional calculations [10] deviates by $10^{-5}$ a.u. In this Table II we also show the convergence with increasing number of points. The change from 625 to 1369 points just leads to changes of a few $10^{-7}$ a.u. only.

One favourable point of the FEM is that it is based on a variational principle, therefore we always get convergence for the total energy with increasing number of points and always from above. This we found in all cases we calculated so far. In the case of the finite difference method the convergence usually is reached from above, but in a few cases it converges from below [34].

The results for the system $\mathrm{BH}$ show a disagreement with the LCAO method [11] at the $10^{-4}$ a.u. level but also a difference of $10^{5}$ a.u. in comparison to the FDM method [14] which is not quite clear to us. The convergence pattern with increasing number of points definitely shows that our calculations are at least one order of magnitude better than the FDM results of Laaksonen et al. [14].

The same statement holds true in the comparison for $\mathrm{N}_{2}$ in Table III. The error in the LCAO calculation [10] is already $10^{3}$ a.u. and the comparison with Laaksonen results shows a disagreement at the $10^{5}$ a.u. level. We do not want to overstress this small disagreement but it is a very clear indication that the FEM method is able to get very accurate results.

The results for $\mathrm{CO}$ are quite similar to those of $\mathrm{N}_{2}$. Again there is a disagreement of the total energy at the $10^{-5} \mathrm{a} . \mathrm{u}$. level with the results of Laaksonen et al. [14]. The change of the total energy when going from 1369 to 2401 grid points is of about $2 \times 10^{-6}$ a.u. Therefore we expect our results to be accurate at a level of $10^{-6}$ a.u.

Thus the FEM proves to be a very powerful tool to achieve very accurate results also for the solution of the HartreeFock equations for diatomic molecules which are unprecedented. The stability of the method is insured by the fact that the FEM is based on a variational principle which is not the case for the FDM. The possibility of using adapted point distributions makes the method very effective. Making practical use of this statement it may be possible to use the FEM also for solution of 3-dimensional quantum mechanical problems within reasonable computer time.
Table III. Total energy and eigenvalues for the diatomic molecules $\mathrm{N}_{2}$ and $\mathrm{CO}$ calculated with the FEM. Comparison is given with the results from FDM and LCAO basis set expansion methods

\begin{tabular}{lcccc}
\hline$N_{2}$ & & & & \\
$R$ & This work & Ref. [14] & Ref. [11] & \\
$E_{\mathrm{toi}}$ & -108.993826 & -108.993808 & -108.9928 & \\
$\varepsilon\left(1 \sigma_{\mathrm{g}}\right)$ & -15.681867 & -15.681862 & -15.6820 & \\
$\varepsilon\left(1 \sigma_{\mathrm{u}}\right)$ & -15.678252 & -15.678246 & -15.6783 & \\
$\varepsilon\left(2 \sigma_{\mathrm{g}}\right)$ & -1.473423 & -1.473401 & -1.4736 & \\
$\varepsilon\left(2 \sigma_{\mathrm{u}}\right)$ & -0.778077 & -0.778075 & -0.7780 & \\
$\varepsilon\left(3 \sigma_{\mathrm{g}}\right)$ & -0.634793 & -0.634784 & -0.6350 & \\
$\varepsilon\left(1 \pi_{\mathrm{u}}^{\mathrm{g}}\right)$ & -0.615625 & -0.615611 & -0.6154 & \\
$C O$ & & & & \\
& & & & \\
Points & This work & This work & Ref. [14] & Ref. [12] \\
$R$ & 1369 & 2401 & & \\
$E_{\mathrm{tot}}$ & -112.732 & 2.132 & 2.132 & 2.132 \\
$\varepsilon(1 \sigma)$ & -20.664521 & -112.790907 & -112.79095 & -112.6986 \\
$\varepsilon(2 \sigma)$ & -11.360051 & -11.360051 & -11.360055 & -11.3945 \\
$\varepsilon(3 \sigma)$ & -1.521490 & -1.521490 & -1.521491 & -1.5665 \\
$\varepsilon(4 \sigma)$ & -0.804530 & -0.804530 & -0.804531 & -0.8006 \\
$\varepsilon(5 \sigma)$ & -0.640361 & -0.640361 & -0.640362 & -0.6493 \\
$\varepsilon(1 \pi)$ & -0.554923 & -0.554923 & -0.554925 & -0.5594 \\
\hline
\end{tabular}

\section{Acknowledgement}

This work was supported by the Swedish Natural Science Research Council, NORDITA and Otto-Braun-Stipendienfonds. Computing time at CRAY X-MP/48 from National Supercomputer Centre at University of Linköping is gratefully acknowledged.

\section{References}

1. Hartree, D. R., The Calculation of Atomic Structures, Wiley, New York (1957)

2. Herman, F. and Skillman, S., Atomic Structure Calculation, PrenticeHall, Englewood Cliffs (1963).

3. Kim, Y. K.. Phys. Rev. 154, 17 (1967); 159, 190 (1967).

4. Froese-Fischer, C., The Hartree-Fock Method for Atoms, Wiley, New York (1977).

5. Desclaux, J. P., Comp. Phys. Comm. 9, 139 (1975).

6. Grant, I. P., McKenzie, B. J., Norrington, P. H., Mayers, D. F. and Pyper, N. C.. Comp. Phys. Comm. 21, 207 (1980).

7. Lindgren, I. and Morrison, J., Atomic Many Body Theory, Springer Series in Chemical Physics, 13, Springer, Belin (1982).

8. Salomonson, S. and Öster, P., Phys., Rev. A40, 5548 (1989).

9. Schaefer III, H. F., The electronic structure of atoms and molecules, Addison and Wesley, Reading (1972).

10. Cade, P. E. and Huo, W. M., J. Chem. Phys. 47, 614 (1967).

11. Cade, P. E., Sales, K. D. and Wahl, A. C., J. Chem. Phys. 44, 1973 (1966).

12. Rose, J., Shibuya, T. and McKoy, V., J. Chem. Phys. 58, 74 (1973).

13. Wilson, S., Basis Sets, in Ab Initio Methods in Quantum Chemistry Part I (Edited by K. P. Lawley) Wiley, New York (1987).

14. Laaksonen, L., Sundholm, D. and Pyykkö, P., Comp. Phys. Rep. 4, 313 (1986).

15. Sundholm, D., Olsen, J., Malmquist, P.-A. and Roos, B. O., in: Numerical Determination of the Electronic Structure of Atoms, Diatomic and Polyatomic Molecules (Edited by M. Defranceschi and J. Delhalle) Kluwer, Dortrecht (1989), pp. 329-334.

16. Heinemann, D., Fricke, B. and Kolb, D., Phys. Rev. A38, 4998 (1988).

17. McCullough, E. A., Comp. Phys. Rep. 4, 267 (1986).

18. Schulze, W. and Kolb, D., Chem. Phys. Lett. 122, 271 (1985).

19. Heinemann, D., Kolb, D. and Fricke, B., Chem. Phys. Lett. 137, 180 1987).

20. Heinemann, D., Fricke, B. and Kolb. D., Chem. Phys. Lett. 145, 125 (1988). 
21. Heinemann, D., Rosén, A. and Fricke, B., Chem. Phys. Lett. 166, 627 (1990).

22. Laaksonen, L., Pyykkö, P. and Sundholm, D., Chem. Phys. Lett. 96, I (1983).

23. Slater, J, C., Quantum Theory of Atomic Structure Vol. I, McGraw Hill, New York 1960), p. 486.

24. Askar, A., Cakmak, A. and Rabitz, H., Chem, Phys. 33, 267 (1978).

25. Duff, M., Rabitz, H., Askar, A., Cakmak, A. and Ablowitz, J., Chem Phys. 72, 1543 (1980).

26. Kashiev, M. S., Vinitsky, S. I. and Vukajlovic, F. R., Phys. Rev. A22, 557 (1980).

27. Ford, W. K. and Levin, F. S., Phys. Rev. A29, 43 (1984).
28. Mishra, M., Linderberg, J. and Öhrn, Y., Chem. Phys. Lett. 111, 439 (1984).

29. Levin, F. S. and Shertzer, J., Phys. Rev. A32, 3285 (1985).

30. Jaquet, R., Theor. Chim. Acta 71, 425 (1987).

31. Johnson, W. R., Blundell, S. A. and Sapirstein, J., Phys. Rev. A37, 307 (1988).

32. Tulkki, J., Ref. [94] in Ref. [14].

33. Tulkki, J., Ref. [5] in: Ref. [34].

34. Pyykkö, P., Diercksen, G. H. F., Müller-Plathe, F. and Laaksonen, L., Chem. Phys. Lett. 134, 575 (1987).

35. Floves, J. R., Clementi, E. and Sonnad, V., Chem. Phys. Lett. 163, 198 (1989). 